\title{
High expression of SEZ6L2 as an independent prognostic Indicator in thyroid carcinoma
}

\author{
Xue Luo ${ }^{1,2}$, Xinghong Chen ${ }^{1}$, Song Chen ${ }^{3}$, Qingjun Gao ${ }^{2}$, Huifang Yang ${ }^{1}$, Daiwei Zhao ${ }^{1,4}$ \\ ${ }^{1}$ Clinical Medical College, Guizhou Medical University, Guiyang, China; ${ }^{2}$ Department of Thyroid Surgery, Affiliated Hospital of Guizhou Medical \\ University, Guiyang, China; ${ }^{3}$ Department of Thyroid Surgery, Jinyang Hospital Affiliated to Guizhou Medical University, Guiyang, China; \\ ${ }^{4}$ Department of General Surgery, the Second People's Hospital of Guizhou Province, Guiyang, China \\ Contributions: (I) Conception and design: X Luo, D Zhao; (II) Administrative support: X Luo, X Chen, H Yang; (III) Provision of study materials \\ or patients: X Luo, X Chen, Q Gao, D Zhao; (IV) Collection and assembly of data: X Luo, X Chen, Q Gao, D Zhao; (V) Data analysis and \\ interpretation: X Chen, S Chen; (VI) Manuscript writing: All authors; (VII) Final approval of manuscript: All authors. \\ Correspondence to: Daiwei Zhao. Guizhou Medical University, Guiyang, China; Department of General Surgery, The Second People's Hospital of \\ Guizhou Province, Guiyang, China. Email: 99276855@qq.com.
}

Background Seizure-related 6 homolog (mouse)-like 2 (SEZ6L2) is a type 1 transmembrane protein that is primarily expressed in the brain. In recent reports, SEZ6L2 has been found to be overexpressed in some cancers and can drive the progression of tumors. However, its function and mechanism in thyroid cancer remain unclear.

Methods: In this article,we searched for the SEZ6L2 expressions in Pan-cancer on TCGA (The Cancer Genome Atlas) and evaluated these data using the TIMER2 method. Then, the immunohistochemical score (IHC score) of SEZ6L2 in cancer tissue was collected in human protein mapping (HPA) data. And we used CIBERSORT to assess the association between the levels of SEZ6L2 expression and the number of various immune cells in papillary thyroid carcinoma (PTC) tissue. Finally, Gene Expression Omnibus (GEO) analyses, real-time quantitative polymerase chain reaction (qRT-PCR) of tissues, and immunohistochemical staining were used to detect the result.

Results: The article illustrated that a large number of cancers had a higher expression of SEZ6L2 compared to the control tissues. And the immunohistochemical score (IHC score) of SEZ6L2 in cancer tissue was considerably elevated compared to that in normal tissues SEZ6L2 was elevated in thyroid carcinoma (THCA) tissue, besides, GEO analyses, qRT-PCR of tissues, and immunohistochemical staining were conducted to test the results. Finally, the Kaplan-Meier survival analysis illustrated that the increased expression of SEZ6L2 was correlated with a dismal prognosis-higher SEZ6L2 is associated with shorter survival. And the univariate analysis illustrated that T stage, SEZ6L2 and Pathologic stage were related to the overall survival (OS), multivariate analysis stated that elevated expression of SEZ6L2 was an independent risk factor that affected progression-free interval $(\mathrm{PFI})(\mathrm{P}<0.05)$. Consequently, we found that the expression of SEZ6L2 was correlated with tumor-infiltrating immune cells by TIMER.

Conclusions: SEZ6L2 was upregulated in patients with THCA and that increased expression of SEZ6L2 was related with clinical progression and was regarded as an independent risk factor for PFI. In THCA patients, the expression of SEZ6L2 could be a significant prognostic factor, which is expected to be a prospective biomarker for THCA in the future.

Keywords: Seizure-related 6 homolog (mouse)-like 2 (SEZ6L2); thyroid carcinoma (THCA); the cancer genome atlas; diagnosis; prognosis

Submitted Dec 14, 2021. Accepted for publication Feb 18, 2022.

doi: $10.21037 /$ gs-22-37

View this article at: https://dx.doi.org/10.21037/gs-22-37 


\section{Introduction}

Thyroid carcinoma (THCA) has been identified as the most commonly occurring type of endocrine malignancy. As indicated in the recent global cancer report, the number of diagnosed THCA patients in 2020 reached 586,000, representing 11 out of every 36 recently diagnosed malignancies worldwide (1). As the most common type of THCA, papillary thyroid carcinoma (PTC) accounts for almost $85-90 \%$ of all cases (2). PTC usually progresses slowly. Furthermore, with standard therapy, PTC frequently displayed an excellent prognosis, with a $93 \% 10$-year survival rate (3). Nevertheless, more than $30 \%$ of cases of THCA displayed an increased propensity of recurrence or early lymph node metastasis (4). As a result, it is critical to find reliable and precise biomarkers for both the diagnosis and the prognosis of THCA.

Seizure-related 6 homolog (mouse)-like 2 (SEZ6L2) is a type 1 transmembrane protein that is primarily expressed in the brain and is affiliated with the seizure-related gene 6 (SEZ6) family, which comprises SEZ6L2, SEZ6L, and SEZ6. Members of the SEZ6 family have primarily been reported in a variety of psychiatric and neurodevelopmental illnesses, including bipolar disorder, epilepsy, intellectual impairment, schizophrenia, and autism (5-12). SEZ6L2 has been demonstrated to modulate differentiation and neurogenesis, as well as to regulate the modulates $\alpha$ amino-3-hydroxy-5-methyl-4-isoxazole propionic acidadducin (AMPA-ADD) signal transmission by controlling phosphorylation of adducin $(13,14)$. Furthermore, a recent research report found the overexpression of AMPA-ADD in lung tumors, which suggests that AMPA-ADD can be used as a potential lung cancer prognostic biomarker (15). SEZ6L2 has been found to be overexpressed in some cancers and can drive the progression of tumors. An et al. found that the up-modulation of SEZ6L2 was strongly associated with the dismal prognosis in CRC patients (16). Wang et al. demonstrated a correlation between the elevated expression of SEZ6L2 protein and tumor size, TNM stage, and tumor number, in hepatocellular carcinoma (HCC) patients. Overexpression of SEZ6L2 was strongly related to the dismal OS and disease-free survival. Additionally, SEZ6L2 was demonstrated to be an independent prognostic indicator for HCC patients' survival (17). And it is indicated that the expression of SEZ6L2 can drive the growth of tumor by mitochondriarelated proteins. Besides, it's also prevents phosphorylation of adducin and neuritogenesis. However, its specific functions in THCA remain unknown. The aim of this research was to examine the expression of SEZ6L2 and its function in patients with THCA. We present the following article in accordance with the REMARK reporting checklist (available at https://gs.amegroups.com/article/ view/10.21037/gs-22-37/rc).

\section{Methods}

\section{Patient data sets}

Patient data sets mRNA expression data (510 samples, Workflow Type: HTSeq-FPKM) and related clinical information were downloaded from the TCGA database (https://portal.gdc.cancer.gov). A total of 510 patients with THCA with the corresponding clinical features were collected in this study. We used the online human protein mapping (HPA) portal website (https://www.proteinatlas. or) to. By inputting the word SEZ6L2 into the Tissue Atlas and Pathology Atlas modules, protein expression data and histochemical staining images of different human normal tissues and tumor tissues were obtained. We standardized the data source according to the product of cell staining intensity, where scores of $0,1,2$, and 3 meant not detected, low, medium, and high, respectively, and according to the number of positive cell expression scores, where $0,1,2$, and 4 points were given when the number of positive cell expression was none, $<25 \%$, $<75 \%$, and $>75 \%$, respectively. The two integrals were added together to obtain the immunohistochemical score. If there were multiple data in the same tissue, the mean value was taken to compare the immunohistochemical score of normal tissue and tumor tissue according to tumor type, and the histogram was drawn.

\section{Sample collection}

THCA and adjacent tissues were enrolled from 40 patients of Guizhou Medical University, immediately stored in liquid nitrogen, and keept at $-80^{\circ} \mathrm{C}$. All procedures performed in this study were in accordance with the Declaration of Helsinki (as revised in 2013). The study was approved by Ethics Committee of Human Trials, Guizhou Medical University [No. 245(2021)] and informed consent was taken from all the patients.

\section{Quantitative real-time PCR of tissues}

Total RNA was extracted from normal thyroid and THCA 
tissue specimens using TRIzol reagent (Invitrogen, Thermo Fisher Scientific, Shanghai, China) on the basis of the manufacturer's instructions. RNA was reversely

RNA was transcribed into cDNA using the Transcription First Strand cDNA synthesis kit (Takara, Dalian, Liaoning, China). Quantitative real-time PCR (qRT-PCR) analyses were quantified with SYBR ${ }^{\circledR}$ Green (Takara). As an internal reference, the hGAPDH was used to calculating the expression of SEZ6L2 based on the $2^{-\Delta \Delta c t}$ method. The real-time quantitative polymerase chain reaction (qRT-PCR) primers used in the present study were as follows: SEZ6L2 forward primer, 5'-ATGAAGCTGGGGATACGC-3'; SEZ6L2 reverse primer, 5'-CCTCGTGGGATAGGGAGA-3'; hGAPDH forward primer, 5'-GGACCTGACCTGCCGTCTAG-3'; and hGAPDH reverse primer, 5'-GTAGCCCAGGATGC CCTT-3'.

\section{Immunobistochemical staining}

Immunohistochemical staining was used to determine the expression of SEZ6L2 in the tissues. Paraffin slices of cancer tissues were taken from the Pathology Department of Guizhou Medical University Hospital, dewaxed with xylene, and hydrated with gradient alcohol (anhydrous ethanol, $95 \%$ ethanol, $80 \%$ ethanol, $70 \%$ ethanol, and $50 \%$ ethanol for 5 minutes each). The tissues were boiled in EDTA solution for 10 minutes and cooled to room temperature, then were washed with PBS. Goat serum working fluid was closed for 30 minutes at $37^{\circ} \mathrm{C}$. Thereafter, the tissues were probed with $50 \mu \mathrm{L}$ of $S E Z 6 L 2$ primary antibody (AF4916, 1:50, R\&D Systems), and then reprobed with $50 \mu \mathrm{L}$ of secondary antibody donkey anti-sheep IgG (a21060, 1:500, Abbkine) for 30 minutes at $37^{\circ} \mathrm{C}$. PBS washing was followed by DAB development for 3 minutes. The tissues were then washed under tap water for 10 minutes, and then the tissues were counterstained with hematoxylin for 20 seconds, differentiated with hydrochloric acid alcohol, gradient alcohol dehydrated, xylene transparentized, and neutral gum sealed before the paraffin slices were observed under a microscope.

\section{Statistical analysis}

Box plots were used to evaluate the level of SEZ6L2 expression in THCA patients. The cut-off value of SEZ6L2 expression was chose as the median method of gene expression. Wilcoxon signed-rank test and logistic regression were used to analyze the association between clinical features and SEZ6L2 expression in THCA. According to the Kaplan-Meier analysis, we compared the progression-free interval (PFI) between the low and high SEZ6L2 expression groups by using the $\mathrm{P}$ value determined in the log-rank test. A received operating characteristic (ROC) curve was adopted to assess the diagnostic value of SEZ6L2 expression, using the area under the ROC curve as the diagnostic value. We conducted statistical analysis and visualization of RNA SEQ data and clinical data of TCGH in TCGA through $\mathrm{R}$ language, then used univariate Cox analysis to filtrate potential prognostic factors, and multivariate Cox analysis to verify the influence of SEZ6L2 expression on survival as well as other clinical variables. An alignment chart was constructed to predict 3-, 5- and 10 -year THCA survival by combining the expression value of SEZ6L2 with clinical variables. In order to test the results, we selected datasets containing both PTC and normal tissues in the Gene Expression Omnibus (GEO) database. The following three microarray datasets were downloaded from the National Center for Biotechnology Information GEO database (http://www.ncbi.nlm.nih.gov/ geo): GSE129562 (P=3.1e-04), GSE65144 (P=6.4e-06), and GSE35570 $(\mathrm{P}=1.9 \mathrm{e}-07)$. The expression level of SEZ6L2 in patients with THCA and normal individuals was further validated in the TIMER database (https://cistrome. shinyapps.io/timer/). All statistical analyses were performed using R statistical software (version 3.6.3), SPSS software (version 24.0) or GSVA (version1.34.0). A P value less than 0.05 was considered as statistically significant.

\section{Results}

\section{Analysis of gene expression data}

The TIMER2 technique was used to determine the expression of SEZ6L2 in various kinds of cancer data retrieved from TCGA. As illustrated in Figure 1A, the expression of SEZ6L2 was significantly elevated as opposed to the control tissues $(\mathrm{P}<0.05)$ of the following tumor types: lung squamous cell carcinoma (LUSC), uterine corpus endometrial carcinoma (UCEC), head and neck squamous cell carcinoma (HNSC), stomach adenocarcinoma (STAD), cervical squamous cell carcinoma, endocervical adenocarcinoma esophageal carcinoma (CESC), liver hepatocellular carcinoma (LIHC), rectum adenocarcinoma (READ), cholangiocarcinoma (CHOL), colon adenocarcinoma (COAD), pheochromocytoma and paraganglioma (PCPG), breast invasive carcinoma (BRCA), esophageal carcinoma 


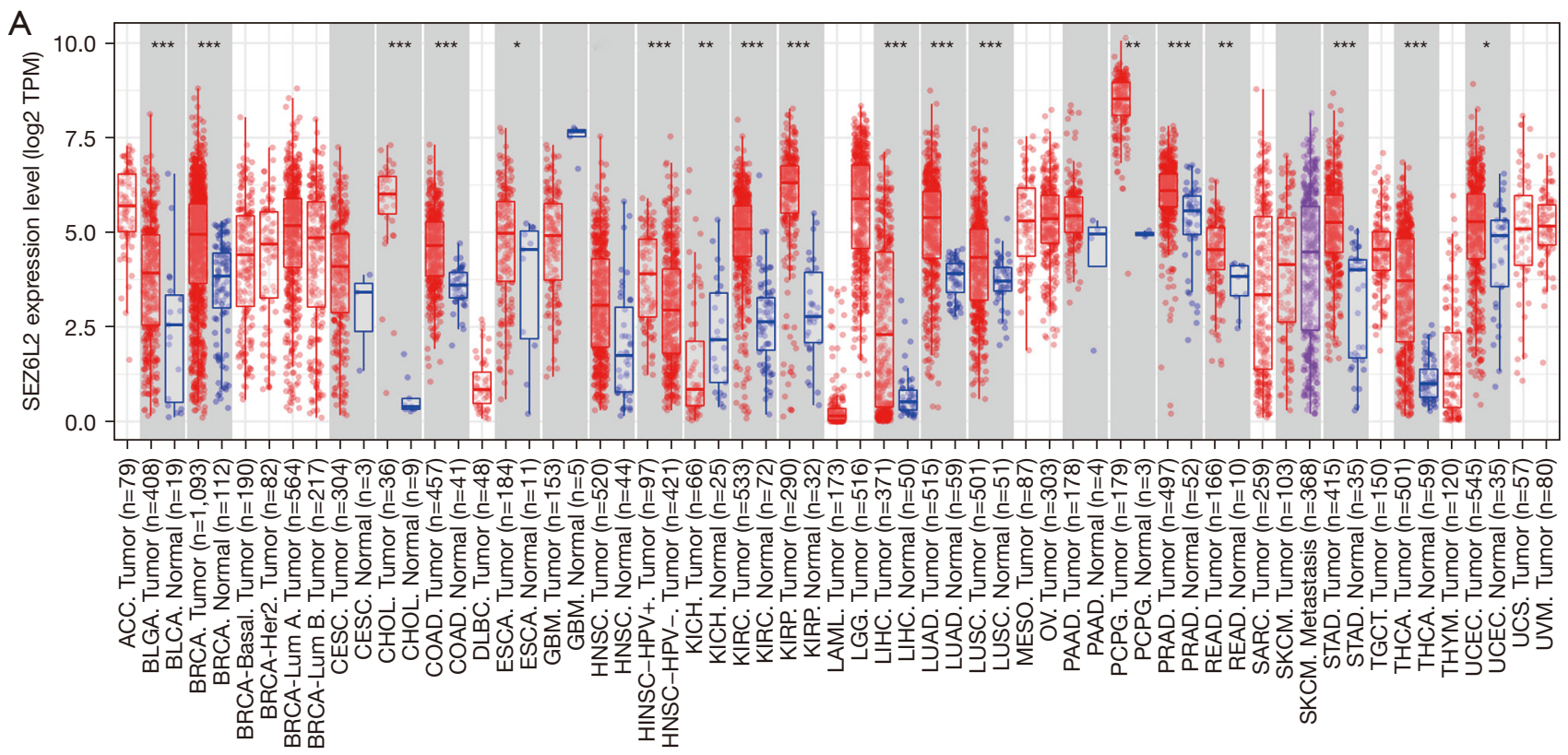

B

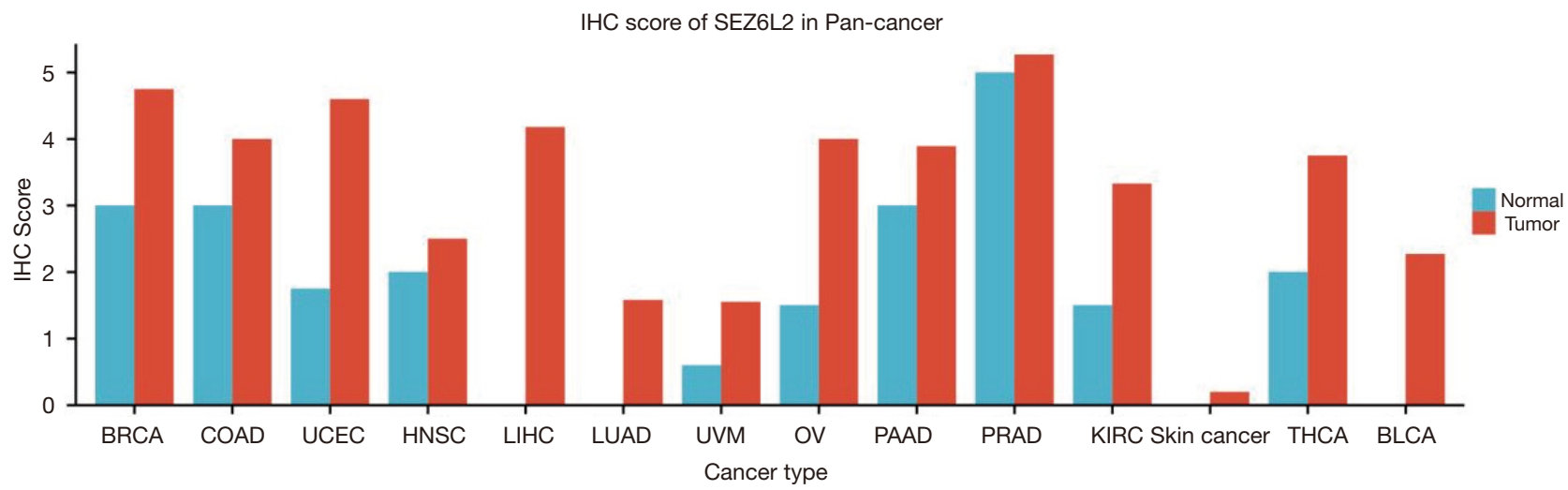

Figure 1 SEZ6L2 expression in Pan-cancer. (A) Comparison of SEZ6L2 expression between different cancerous and paraneoplastic tissues. (B) immunohistochemical score of SEZ6L2 in Pan-cancer. * $\mathrm{P}<0.05$; ** $\mathrm{P}<0.01$; *** $\mathrm{P}<0.001$. ACC, adrenocortical carcinoma; BLCA, bladder urothelial carcinoma; BRCA, breast invasive carcinoma; CESC, cervical squamous cell carcinoma and endocervical adenocarcinoma; CHOL, cholangiocarcinoma; COAD, colon adenocarcinoma; DLBC, lymphoid neoplasm diffuse large B-cell lymphoma; ESCA, esophageal carcinoma; GBM, glioblastoma multiforme; HNSC, head and neck squamous cell carcinoma; KICH, kidney chromophobe; KIRC, kidney renal clear cell carcinoma; KIRP, kidney renal papillary cell carcinoma; LAML, acute myeloid leukemia; LGG, brain lower grade glioma; LIHC, liver hepatocellular carcinoma; LUAD, lung adenocarcinoma; LUSC, lung squamous cell carcinoma; MESO, mesothelioma; OV, ovarian serous cystadenocarcinoma; PAAD, pancreatic adenocarcinoma; PCPG, pheochromocytoma and paraganglioma; PRAD, prostate adenocarcinoma; READ, rectum adenocarcinoma; SARC, sarcoma; SKCM, skin cutaneous melanoma; STAD, stomach adenocarcinoma; TGCT, testicular germ cell tumors; THCA, thyroid carcinoma; THYM, thymoma; UCEC, uterine corpus endometrial carcinoma; UCS, Uterine carcinosarcoma; UVM, uveal melanoma.

(ESCA), THCA, lung adenocarcinoma (LUAD), kidney chromophobe (KICH), kidney renal clear cell carcinoma (KIRC), prostate adenocarcinoma (PRAD), glioblastoma multiforme (GBM), kidney renal papillary cell carcinoma
(KIRP), and bladder urothelial carcinoma (BLCA) (Table 1).

Then, we collected the data about the normal and tumor tissue comparisons in the HPA database. The immunohistochemical score (IHC score) of SEZ6L2 in 
Table 131 types of human cancers employed in research

\begin{tabular}{|c|c|}
\hline Abbreviation & Full name \\
\hline ACC & Adrenocortical carcinoma \\
\hline BLCA & Bladder urothelial carcinoma \\
\hline BRCA & Breast invasive carcinoma \\
\hline CESC & $\begin{array}{l}\text { Cervical squamous cell carcinoma and } \\
\text { endocervical adenocarcinoma }\end{array}$ \\
\hline $\mathrm{CHOL}$ & Cholangiocarcinoma \\
\hline COAD & Colon adenocarcinoma \\
\hline DLBC & $\begin{array}{l}\text { Lymphoid neoplasm diffuse large B-cell } \\
\text { lymphoma }\end{array}$ \\
\hline ESCA & Esophageal carcinoma \\
\hline HNSC & Head and neck squamous cell carcinoma \\
\hline $\mathrm{KICH}$ & Kidney chromophobe \\
\hline KIRC & Kidney renal clear cell carcinoma \\
\hline KIRP & Kidney renal papillary cell carcinoma \\
\hline LAML & Acute myeloid leukemia \\
\hline LGG & Brain lower grade glioma \\
\hline $\mathrm{LIHC}$ & Liver hepatocellular carcinoma \\
\hline LUAD & Lung adenocarcinoma \\
\hline LUSC & Lung squamous cell carcinoma \\
\hline MESO & Mesothelioma \\
\hline OV & Ovarian serous cystadenocarcinoma \\
\hline PAAD & Pancreatic adenocarcinoma \\
\hline PCPG & Pheochromocytoma and paraganglioma \\
\hline PRAD & Prostate adenocarcinoma \\
\hline READ & Rectum adenocarcinoma \\
\hline SARC & Sarcoma \\
\hline SKCM & Skin cutaneous melanoma \\
\hline STAD & Stomach adenocarcinoma \\
\hline TGCT & Testicular germ cell tumors \\
\hline THCA & Thyroid carcinoma \\
\hline THYM & Thymoma \\
\hline UCEC & Uterine corpus endometrial carcinoma \\
\hline UCS & Uterine carcinosarcoma \\
\hline UVM & Uveal melanoma \\
\hline
\end{tabular}

cancer tissue was considerably elevated compared to that in normal tissues among 14 tumors, including BRCA, COAD, UCEC, HNSC, UVM, OV, PAAD, PRAD, KIRC, THCA, and BLCA, as illustrated in Figure $1 B$.

\section{Patients' baseline characteristics}

Data from 510 THCA patients who met the criteria for the necessary clinical data was retrieved in June 2021 from TCGA. Table 2 displays a detailed list of the clinical characteristics. The 510 patients were classified into two groups according to their median SEZ6L2 expression value. The group with low expression of SEZ6L2 had 304 cases, and the higher group had 206 cases. In terms of T stage, the low expression group included 99 patients (19.4\%) at T1 stage, 100 patients $(19.6 \%)$ at T2 stage, 88 patients $(17.3 \%)$ at T3 stage, and 15 patients (2.9\%) at T4 stage. In the high expression group, 44 patients $(8.6 \%)$ were T1 stage, 67 patients $(13.1 \%)$ were $\mathrm{T} 2$ stage, 87 patients $(17.1 \%)$ were T3 stage, and 8 patients (1.6\%) were T4 stage. As for the $\mathrm{N}$ stage, the low expression group included 141 patients $(27.6 \%)$ at $\mathrm{N} 0$ stage and 125 patients $(24.5 \%)$ at $\mathrm{N} 1$ stage. The high expression group contained 88 patients $(17.3 \%)$ at $\mathrm{N} 0$ stage and 106 patients $(20.8 \%)$ at $\mathrm{N} 1$ stage $(\mathrm{P}<0.05)$. In addition, in the low expression group 166 patients were M0 stage $(32.5 \%)$ and 6 patients were M1 stage (1.2\%), while the high expression group contained 120 patients at M0 stage $(23.5 \%)$ and 3 patients at M1 stage $(0.6 \%)$.

\section{High SEZ6L2 expression in THCA}

We examined the level of SEZ6L2 expression in normal thyroid tissues and in tumor specimens to ascertain the degree of SEZ6L2 expression in THCA patients. The findings indicated that $S E Z 6 L 2$ gene expression was considerably elevated in THCA tissues ( $\mathrm{P}=1 \mathrm{e}-23)$ compared to that in normal tissues (Figure 2A). These findings were confirmed in THCA tissues as well as in paired normal thyroid tissues $(\mathrm{P}=3.8 \mathrm{e}-10)$ (Figure $2 B)$. Further research examined gene expression data from three GEO cohorts, and these findings corroborated the aforementioned results [GSE129562 ( $\mathrm{P}=3.1 \mathrm{e}-04) ;$ GSE65144 $(\mathrm{P}=6.4 \mathrm{e}-06)$; and GSE35570 ( $\mathrm{P}=1.9 \mathrm{e}-07)]$ (Figure $2 C-2 E)$. In order to test the results, we did a qRT-PCR of tissues and showed that the 
Table 2 Clinical features of the THCA

\begin{tabular}{lcc}
\hline Characteristic & Low expression of SEZ6L2 (N=304) & High expression of SEZ6L2 (N=206) \\
\hline T stage, $\mathrm{n}(\%)$ & $99(19.4)$ & $44(8.6)$ \\
T1 & $100(19.6)$ & $67(13.1)$ \\
T2 & $88(17.3)$ & $87(17.1)$ \\
T3 & $15(2.9)$ & $8(1.6)$ \\
T4 & & \\
N stage, $n(\%)$ & $141(27.6)$ & $88(17.3)$ \\
N0 & $125(24.5)$ & $106(20.8)$ \\
N1 & & $120(23.5)$ \\
M stage, $n(\%)$ & $166(32.5)$ & $3(0.6)$ \\
M0 & $6(1.2)$ & 0.032 \\
M1 & & \\
\hline
\end{tabular}

THCA, thyroid carcinoma.

level of SEZ6L2 gene expression was considerably elevated in THCA tissues. These findings were validated in THCA tissues and paired normal thyroid tissues $(\mathrm{P}=1.3 \mathrm{e}-07)$ (Figure $2 F$ ). The immunohistochemistry findings also showed that SEZ6L2 expression was significantly elevated in THCA tissues compared to that in normal tissues (Figure 2G,2H). The SEZ6L2 expression in tumor tissues was considerably elevated compared to that in normal tissues in these 14 tumors from the HPA data, including THCA (Figure 2I).

\section{Relationship between SEZ6L2 expression and clinical characteristics}

Table 2 summarizes the association between SEZ6L2 expression and clinical characteristics in THCA patients. Elevated expression of SEZ6L2 was substantially associated with $\mathrm{N}$ classification $(\mathrm{P}=0.026)$, $\mathrm{T}$ classification $(\mathrm{P}=0.046)$ and clinical stage $(\mathrm{P}<0.001)$, as illustrated in Figure 3. With the aid of logistic regression, a univariate analysis illustrated that the SEZ6L2 expression was a categorical dependent variable related to the dismal prognosis in terms of clinical outcomes (Table 3). Elevated expression of SEZ6L2 was considerably correlated with $\mathrm{T}$ classification $[\mathrm{T} 3$ and $\mathrm{T} 4$ vs. $\mathrm{T} 1$ and $\mathrm{T} 2 \mathrm{OR}=1.441 ; 95 \%$ confidence interval $(\mathrm{CI})=1.008-2.065 ; \mathrm{P}=0.046), \mathrm{N}$ classification $(\mathrm{N} 1 v s$. $\mathrm{NO} O R=1.521 ; 95 \% \mathrm{CI}=1.053-2.200 ; \mathrm{P}=0.026)$, and pathologic stage (stage III and IV vs. stage I and II OR
$=1.881 ; 95 \% \mathrm{CI}=1.296-2.745 ; \mathrm{P}<0.001]$. According to the clinical features, multivariate and univariate analysis were finished. Univariate analysis illustrated that $\mathrm{T}$ stage, SEZ6L2 and pathologic stage were related to the overall survival (OS). And multivariate analysis stated that SEZ6L2 is the independent risk for it $(\mathrm{P}<0.05)$ (Table 4).

\section{SEZ6L2 overexpression is an independent risk factor for PFI}

Liu et al. summarised the recommendations for endpoint use for each cancer type. In the case of less severe tumor types such as THCA, the progression-free interval (PFI) and the disease-free interval (DFI) were suggested as measures of tumor response (18). As illustrated in Figure 4, a Kaplan-Meier survival analysis found that the elevated level of SEZ6L2 expression was correlated with a dismal prognosis. Elevated SEZ6L2 expression was demonstrated to have a strong link to a grim prognosis in clinical stage III/IV ( $\mathrm{P}=0.023), \mathrm{N} 1(\mathrm{P} \leq 0.001)$, and T2/T3 $(\mathrm{P}=0.027)$ according to subgroup analysis based on distinct clinical characteristics. PFI was shown to be substantially correlated with increased SEZ6L2 expression, according to a univariate Cox analysis [hazard ratio $(\mathrm{HR})=1.34 ; 95 \%$ CI $=1.16-1.54 ; \mathrm{P}=4.86 \mathrm{e}-05]$. The forest plot of the multivariate Cox regression analysis verified that $S E Z 6 L 2$ gene expression was an independent risk factor that affected the PFI in THCA patients $(\mathrm{P}<0.05)$, as demonstrated in Figure 5 . 

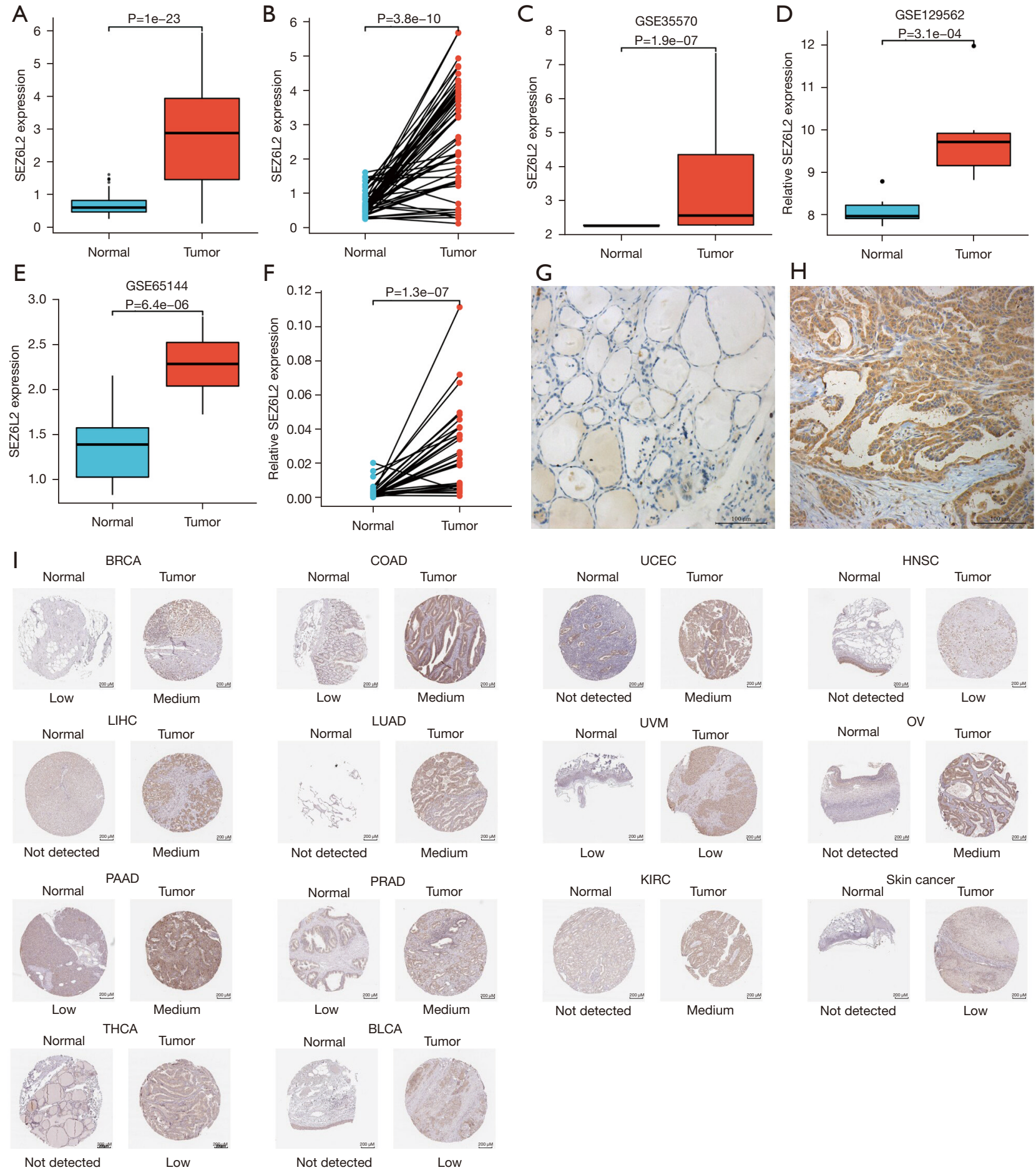

Figure 2 SEZ6L2 expression in PTC tissues. (A) The expression of SEZ6L2 in tumor and normal tissues in TCGA. (B) The expression of SEZ6L2 in paired tissues in TCGA. The expression of SEZ6L2 in normal and tumor tissue in GEO (C-E). The expression of SEZ6L2 in tumor and normal tissues by PCR (F). The expression of SEZ6L2 in normal tissues (G) and THCA tissues (H) by immunohistochemical (scale bar $=100 \mu \mathrm{m}$ ). The expression of SEZ6L2 in normal and tumor tissues in the HPA database (method: SEZ6L2 was combined with antibody, diaminobenzidine showed the color; scale bar =200 $\mu \mathrm{m})(\mathrm{I})$. PTC, papillary thyroid carcinoma; GEO, Gene Expression Omnibus; PCR, polymerase chain reaction; THCA, thyroid carcinoma; HPA, Human Protein Atls. 

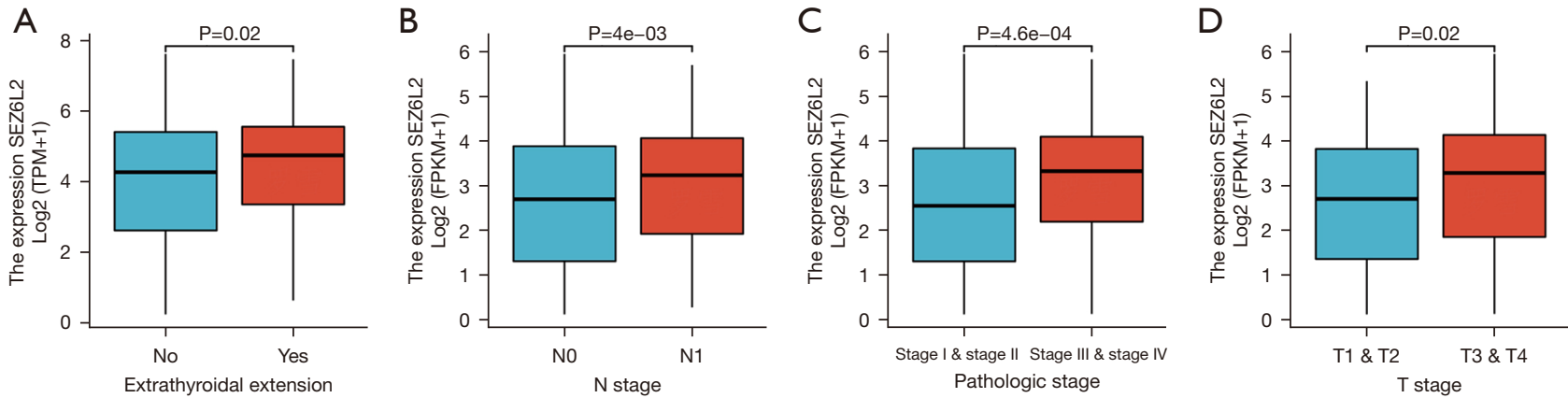

Figure 3 Box plot demonstrating the expression of SEZ6L2 in thyroid cancer patients with various clinical features (A-D).

Table 3 The relationship between SEZ6L2 expression and clinical features analyzed using a logistic regression model

\begin{tabular}{lccc}
\hline Characteristics & Total $(\mathrm{n})$ & Odds ratio (OR) & P \\
\hline T stage (T3 \& T4 vs. T1 \& T2) & 508 & $1.441(1.008-2.065)$ & 0.046 \\
N stage (N1 vs. N0) & 460 & $1.521(1.053-2.200)$ & 0.026 \\
M stage (M1 vs. M0) & 295 & $0.460(0.096-1.778)$ & 0.278 \\
Pathologic stage (Stage III \& Stage IV vs. & 508 & $1.881(1.296-2.745)$ & $<0.001$ \\
Stage I \& Stage II) & & & 0.329 \\
Age (>45 vs. $\leq 45)$ & 510 & $1.189(0.840-1.685)$ & 0.197 \\
Gender (male vs. female) & 510 & $1.294(0.876-1.917)$ & 0.052 \\
Extrathyroidal extension (yes vs. no) & 492 & $1.462(0.997-2.150)$ & \\
\hline
\end{tabular}

Table 4 Multivariate and univariate Cox regression analyses of clinical features related to OS

\begin{tabular}{|c|c|c|c|c|}
\hline Characteristics & \multicolumn{2}{|c|}{ Univariate analysis } & \multicolumn{2}{|c|}{ Multivariate analysis } \\
\hline T stage (T3 \& T4 vs. $\mathrm{T} 1 \&$ $\mathrm{T} 2$ ) & $2.530(1.410-4.540)$ & $0.001^{*}$ & 1.717 (0.869-3.392) & 0.119 \\
\hline $\mathrm{N}$ stage (N1 vs. N0) & $1.578(0.884-2.815)$ & 0.122 & $1.152(0.630-2.103)$ & 0.644 \\
\hline SEZ6L2 (high vs. low) & 1.034 (1.011-1.058) & $0.003^{*}$ & $1.025(1.002-1.049)$ & 0.003 \\
\hline Gender (female vs. male) & $1.361(0.746-2.482)$ & 0.313 & $1.143(0.622-2.101)$ & 0.665 \\
\hline Age (>45 vs. $\leq 45)$ & $1.784(0.889-3.582)$ & 0.103 & $1.145(0.532-2.463)$ & 0.727 \\
\hline
\end{tabular}

${ }^{*}, \mathrm{P}<0.05$. OS, overall survival.

\section{Diagnostic significance of SEZ6L2 expression in THCA}

A ROC curve was used to examine the diagnostic significance of SEZ6L2 expression in patients with THCA. As illustrated in Figure 6A, the AUC was 0.902, indicating that the SEZ6L2 had an excellent diagnostic significance. Based on the subgroup analysis, the AUC values for the diagnostic significance of SEZ6L2 gene expression in various clinical characteristics for PTC were as follows: 0.936 for M0, 0.891 for stage I/II, 0.924 for stage III/IV, 

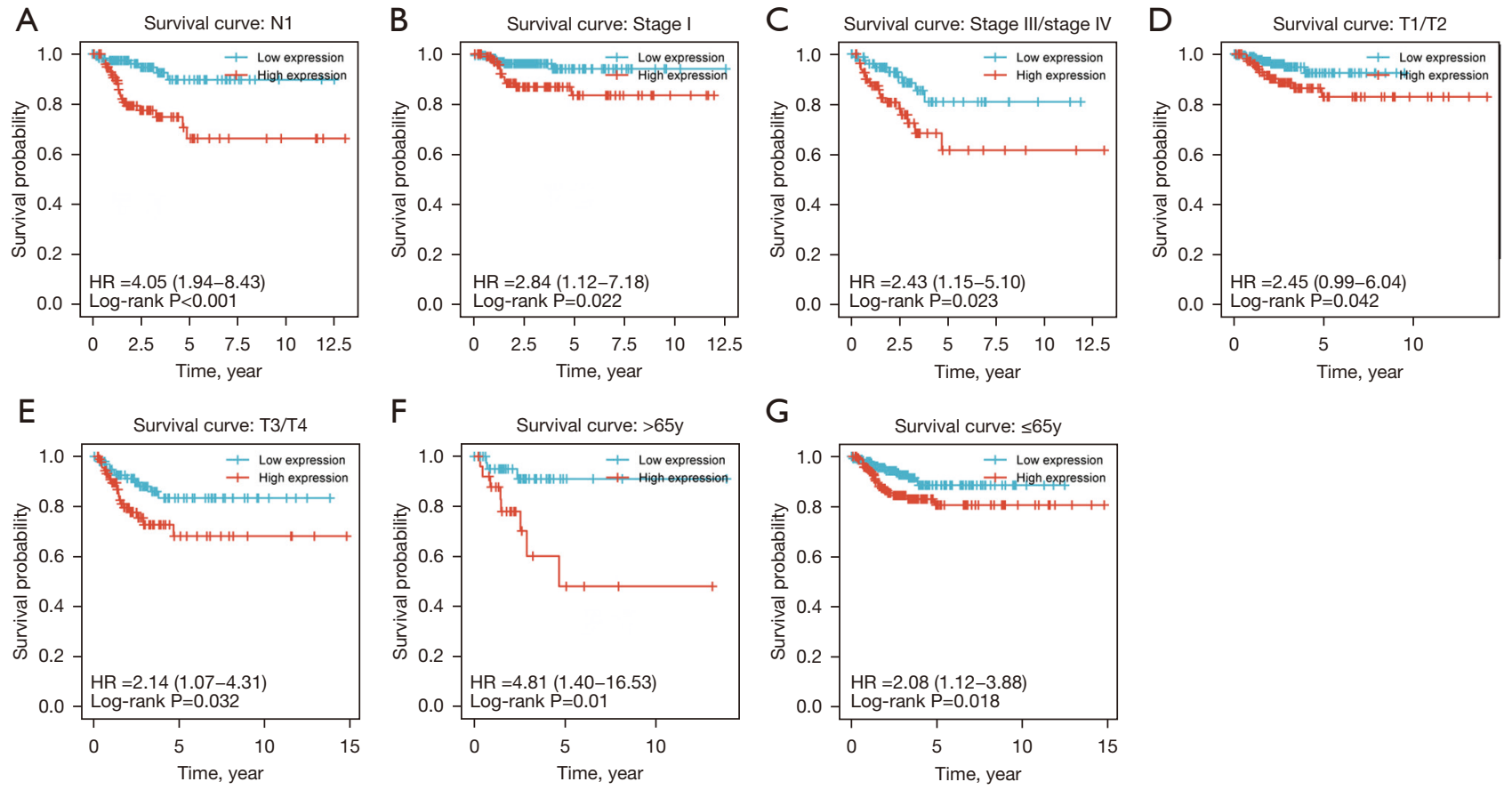

Figure 4 Kaplan-Meier curve for OS in THCA. (A-G) Subgroup analysis for N1, stage I, stage III/stage IV, T1/T2, T3/T4, >65 years, and $\leq 65$ years. OS, overall survival; THCA, thyroid carcinoma.

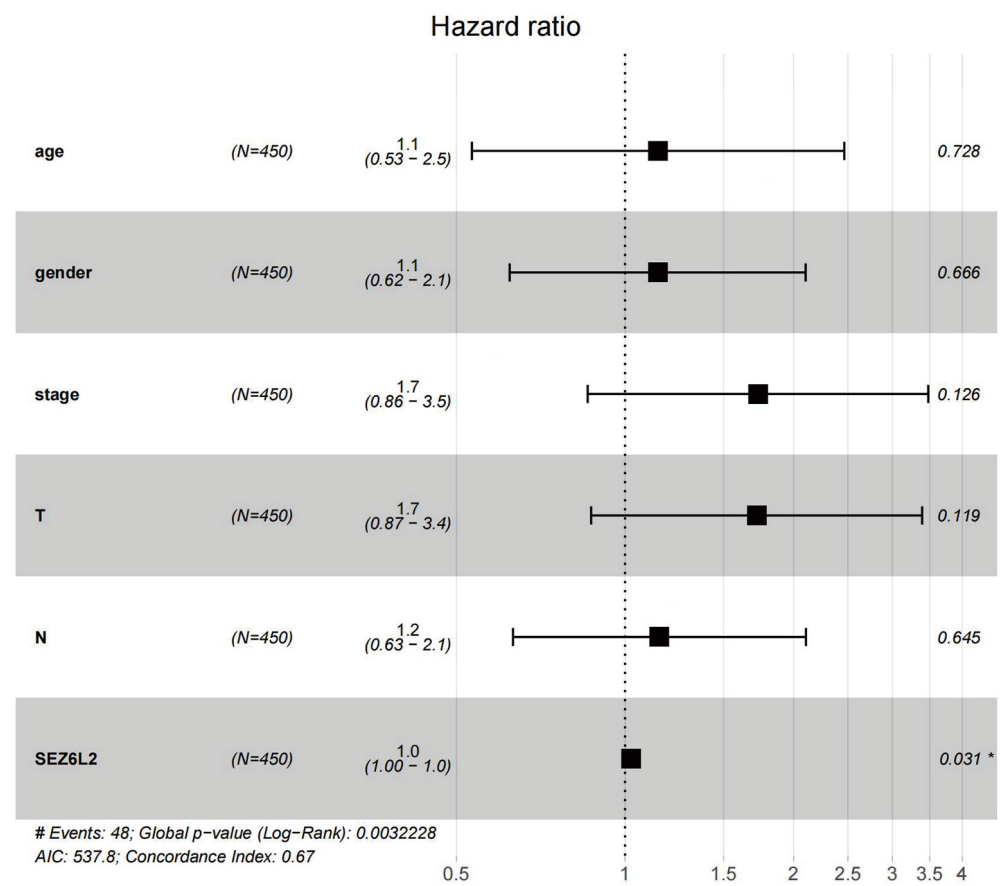

Figure 5 The forest plot illustrating the results of the multivariate Cox regression analysis in THCA. *, P<0.05. THCA, thyroid carcinoma. 

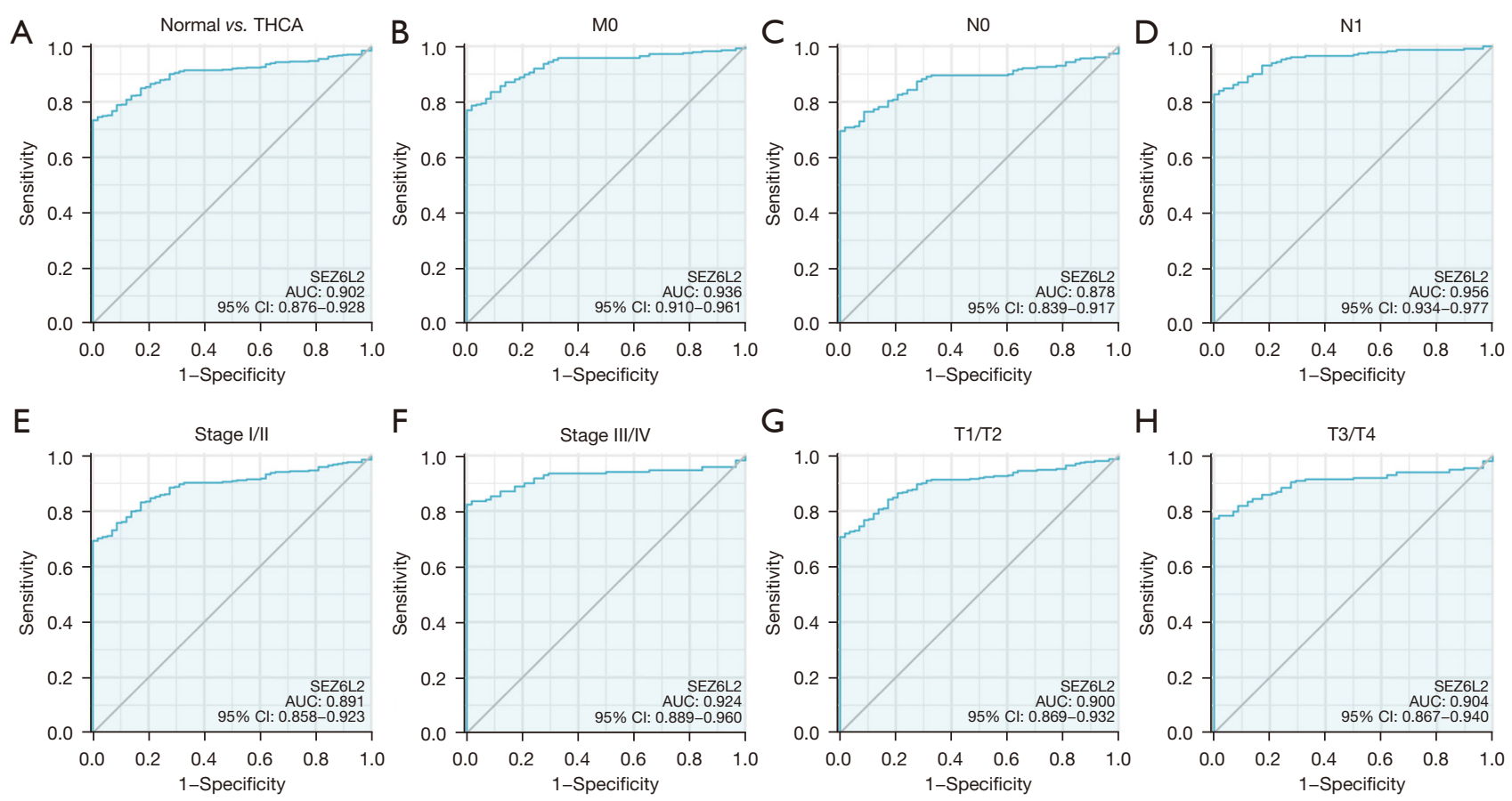

Figure 6 Diagnostic value of SEZ6L2 expression in THCA. (A) ROC curve for SEZ6L2 in normal thyroid tissue and THCA. (B-H) Subgroup analysis for M0, N0, N1, stage I/II, stage III/IV, T1/T2, T3/T4. THCA, thyroid carcinoma; ROC, receiver operating characteristic.

Points
Age
Gender
Pathologic stage
T stage
N stage
SEZ6L2
Total points
Linear predictor
3-year survival probability
5-year survival probability
10-year survival probability

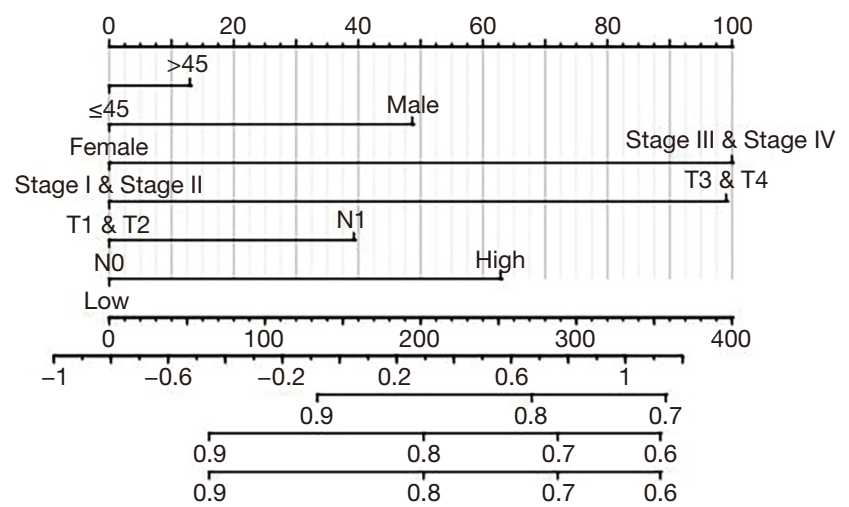

Figure 7 Nomogram anticipating the 3-, 5- and 10-year OS of patients. To estimate the risk, the status of each SEZ6L2 expression value and the clinical feature was determined by sketching a straight line up towards the point axis and looking at the points generated by individual features. This was repeated until the final scores for all variables have been determined. We added up all of the points and plotted the resulting point on the Total Points axis. Subsequently, by drawing a straight line downwards down the risk axis, the 3-, 5-, and 10-year associated survival rates were yielded.

0.900 for T1/T2, and 0.904 for T3/T4 (Figure $6 B-6 H$ ). As a result, a nomogram was developed to anticipate the 3-, 5-, and 10 -year survival rates of patients by integrating the level of SEZ6L2 expression with clinical factors (Figure 7).

\section{Relationship between SEZ6L2 expression and different immune cells}

We used CIBERSORT to assess the association between the levels of SEZ6L2 expression and the number of various 
Table 5 Spearman and Pearson correlation analyses between SEZ6L2 and immune cells

\begin{tabular}{|c|c|c|c|c|c|}
\hline Molecule & Cell & Pearson & $\mathrm{P}$ (Pearson) & Spearman & $\mathrm{P}($ Spearman) \\
\hline SEZ6L2 & B cells & 0.097 & 0.028 & 0.124 & 0.005 \\
\hline SEZ6L2 & CD8 T cells & 0.023 & 0.604 & 0.037 & 0.402 \\
\hline SEZ6L2 & Cytotoxic cells & 0.069 & 0.121 & 0.086 & 0.053 \\
\hline SEZ6L2 & Eosinophils & 0.193 & $<0.001$ & 0.182 & $<0.001$ \\
\hline SEZ6L2 & iDC & 0.309 & $<0.001$ & 0.301 & $<0.001$ \\
\hline SEZ6L2 & Macrophages & 0.347 & $<0.001$ & 0.344 & $<0.001$ \\
\hline SEZ6L2 & Mast cells & 0.287 & $<0.001$ & 0.274 & $<0.001$ \\
\hline SEZ6L2 & NK CD56dim cells & 0.053 & 0.231 & 0.059 & 0.184 \\
\hline SEZ6L2 & NK cells & -0.041 & 0.352 & -0.062 & 0.162 \\
\hline SEZ6L2 & $\mathrm{pDC}$ & -0.017 & 0.704 & -0.027 & 0.546 \\
\hline SEZ6L2 & T cells & 0.133 & 0.003 & 0.161 & $<0.001$ \\
\hline SEZ6L2 & $\mathrm{T}$ helper cells & 0.049 & 0.269 & 0.093 & 0.035 \\
\hline SEZ6L2 & Tcm & 0.046 & 0.301 & 0.050 & 0.257 \\
\hline SEZ6L2 & Tem & 0.195 & $<0.001$ & 0.173 & $<0.001$ \\
\hline SEZ6L2 & TFH & 0.151 & $<0.001$ & 0.190 & $<0.001$ \\
\hline
\end{tabular}

immune cells in PTC tissue in order to further investigate the correlation between the levels of SEZ6L2 expression and the immune response. The findings showed that SEZ6L2 overexpression was associated with a greater proportion of macrophages, treg, DC, neutrophils, aDC, iDC, Mast cells, Th1 cells, Th2 cells, TFH, eosinophils and Tem $(\mathrm{P}<0.05)$, while SEZ6L2 overexpression was associated with a decrease in the proportion of NK cells, Th17 cells, and Tgd. To examine if the expression of SEZ6L2 was associated with the immune cell, Spearman and Pearson analyses of association were performed. As illustrated in Table 5, the expression of SEZ6L2 was associated with aDC, neutrophils, DC, Mast cells, iDC, macrophages, T cells, B cells, Tem, Tfh, Th1 cells, Th2 cells, eosinophils, and TReg [P (Spearman, Pearson) <0.05]. According to the findings, SEZ6L2 expression was strongly associated with innate immune cells (Figure 8).

\section{Discussion}

The Sez6 family comprises three members, Sez6, Sez6L, and SEZ6L2, all of which have been found to have an effect on synapse numbers and dendritic morphology. These genes have also been correlated with a variety of neurological and mental diseases (19). Members of the Sez6 family have been regarded as indicators of dismal prognosis 


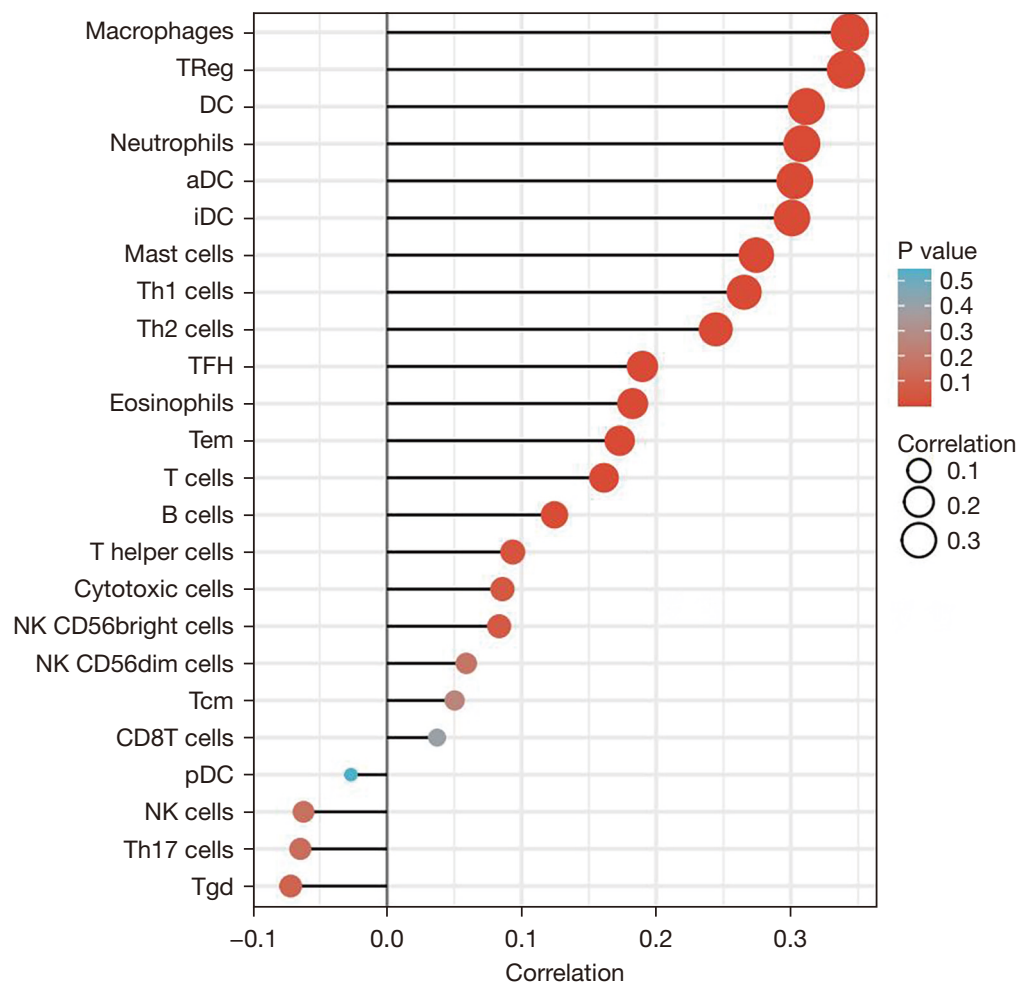

Figure 8 The association between SEZ6L2 and immune cells in THCA. THCA, thyroid carcinoma.

in cancers that are not located in the nervous system (20-23). Wang et al. demonstrated that HCC samples had a greater level of the SEZ6L2 protein expression, which was associated with tumor size, tumor-node-metastasis (TNM) stages, and tumor number. Moreover, overexpression of SEZ6L2 was strongly correlated with the dismal OS and DFS in patients with HCC (17). An et al. illustrated that SEZ6L2 expression was substantially elevated in the tumor tissues of CRC patients as opposed to adjoining normal tissues. They also found that SEZEL2 performs an integral function in the onset, progression, and malignant behavior of tumors (16). Nevertheless, the SEZ6L2 gene and the relationship between the expression levels of the SEZ6L2 gene and therapeutic value in THCA patients remain poorly understood.

As far as we know, ours is the first research report that assesses the association between the expression of SEZ6L2 and clinical parameters in THCA patients. In our research, we searched for the SEZ6L2 expressions among various forms of cancer data present on TCGA and evaluated these data using the TIMER2 method. The results illustrated that a large number of cancers had a higher expression of SEZ6L2 compared to the control tissues. Moreover, the immunohistochemical score (IHC score) of SEZ6L2 in cancer tissue was considerably elevated compared to that in normal tissues of HPA data, including THCA. SEZ6L2 had a high predictive significance in the context of pan-cancer.

Subsequently, we searched for RNA sequencing data in the TCGA, and the findings indicated that SEZ6L2 was elevated in THCA tissue. In addition, we investigated gene expression data from three GEO cohorts, and all of the findings corroborated the conclusions stated above [GSE129562 (P=3.1e-04), GSE65144 ( $\mathrm{P}=6.4 \mathrm{e}-06)$, and GSE35570 $(\mathrm{P}=1.9 \mathrm{e}-07)]$ (Figure $2 C-2 E)$. In order to test the results, we conducted 3 GEO analyses, qRT-PCR of tissues, and immunohistochemical staining, which indicated that SEZ6L2 expression was substantially elevated in THCA tissues compared to the normal tissues. Subsequently, we summarized the association between SEZ6L2 expression and the clinical features of THCA patients by logistic analysis. The elevated expression of SEZ6L2 was correlated with clinical, $\mathrm{T}$, and $\mathrm{N}$ stages. The Kaplan-Meier survival analysis illustrated that the increased expression of SEZ6L2 was correlated with a dismal prognosis. Moreover, the elevated expression of SEZ6L2 was an independent risk factor that affected PFI. Consequently, we used the TIMER 
algorithms to examine the immune cell infiltrations. We found that the expression of SEZ6L2 was correlated with tumor-infiltrating immune cells and was positively correlated with the immune infiltration of macrophages, Treg, DC, neutrophils, aDC, iDC, Mast cells, Th1 cells, Th2 cells, TFH, eosinophils, and Tem.

\section{Conclusions}

We demonstrated that SEZ6L2 was upregulated in patients with THCA and that increased expression of SEZ6L2 was correlated with clinical progression and was regarded as an independent risk factor for PFI. In patients with THCA, the expression of SEZ6L2 could be a significant prognostic factor. However, our research has limitations, since we did not investigate the molecular processes and the relative function of SEZ6L2 in THCA. In view of this, we plan to perform further in-depth mechanistic and functional research on cell lines and mice in the future. Taken together, our data showed that SEZ6L2 is overexpressed in THCA tissue and that SEZ6L2 may be regarded as a major independent prognostic indicator as well as a promising treatment target for THCA.

\section{Acknowledgments}

Funding: The study was supported by the National Natural Science Foundation of China (No. 81860478).

\section{Footnote}

Reporting Checklist: The authors have completed the REMARK reporting checklist. Available at https:// gs.amegroups.com/article/view/10.21037/gs-22-37/rc

Data Sharing Statement: Available at https://gs.amegroups. com/article/view/10.21037/gs-22-37/dss

Conflicts of Interest: All authors have completed the ICMJE uniform disclosure form (available at https://gs.amegroups. com/article/view/10.21037/gs-22-37/coif). The authors have no conflicts of interest to declare.

Ethical Statement: The authors are accountable for all aspects of the work in ensuring that questions related to the accuracy or integrity of any part of the work are appropriately investigated and resolved. All procedures performed in this study were in accordance with the
Declaration of Helsinki (as revised in 2013). The study was approved by Ethics Committee of Human Trials, Guizhou Medical University [No. 245(2021)]_and informed consent was taken from all the patients.

Open Access Statement: This is an Open Access article distributed in accordance with the Creative Commons Attribution-NonCommercial-NoDerivs 4.0 International License (CC BY-NC-ND 4.0), which permits the noncommercial replication and distribution of the article with the strict proviso that no changes or edits are made and the original work is properly cited (including links to both the formal publication through the relevant DOI and the license). See: https://creativecommons.org/licenses/by-nc-nd/4.0/.

\section{References}

1. Sung H, Ferlay J, Siegel RL, et al. Global Cancer Statistics 2020: GLOBOCAN Estimates of Incidence and Mortality Worldwide for 36 Cancers in 185 Countries. CA Cancer J Clin 2021;71:209-49.

2. Kitahara CM, Sosa JA. The changing incidence of thyroid cancer. Nat Rev Endocrinol 2016;12:646-53.

3. Yu QA, Ma DK, Liu KP, et al. Clinicopathologic risk factors for right paraesophageal lymph node metastasis in patients with papillary thyroid carcinoma. J Endocrinol Invest 2018;41:1333-8.

4. Xing M. Molecular pathogenesis and mechanisms of thyroid cancer. Nat Rev Cancer 2013;13:184-99.

5. Chapman NH, Nato AQ Jr, Bernier R, et al. Whole exome sequencing in extended families with autism spectrum disorder implicates four candidate genes. Hum Genet 2015;134:1055-68.

6. Ambalavanan A, Girard SL, Ahn K, et al. De novo variants in sporadic cases of childhood onset schizophrenia. Eur J Hum Genet 2016;24:944-8.

7. Malhotra D, Sebat J. CNVs: harbingers of a rare variant revolution in psychiatric genetics. Cell 2012;148:1223-41.

8. Weiss LA, Shen Y, Korn JM, et al. Association between microdeletion and microduplication at 16p11.2 and autism. N Engl J Med 2008;358:667-75.

9. Kumar RA, Marshall CR, Badner JA, et al. Association and mutation analyses of $16 \mathrm{p} 11.2$ autism candidate genes. PLoS One 2009; 4:e4582.

10. Konyukh M, Delorme R, Chaste P, et al. Variations of the candidate SEZ6L2 gene on Chromosome 16p11.2 in patients with autism spectrum disorders and in human populations. PLoS One 2011;6:e17289. 
11. Xu C, Mullersman JE, Wang L, et al. Polymorphisms in seizure 6-like gene are associated with bipolar disorder I: evidence of gene $\times$ gender interaction. J Affect Disord 2013;145:95-9.

12. Mulley JC, Iona X, Hodgson B, et al. The Role of SeizureRelated SEZ6 as a Susceptibility Gene in Febrile Seizures. Neurol Res Int 2011;2011:917565.

13. Yaguchi H, Yabe I, Takahashi H, et al. SEZ6L2 regulates phosphorylation of ADD and neuritogenesis. Biochem Biophys Res Commun 2017;494:234-41.

14. Boonen M, Staudt C, Gilis F, et al. Cathepsin D and its newly identified transport receptor SEZ6L2 can modulate neurite outgrowth. J Cell Sci 2016;129:557-68.

15. Ishikawa N, Daigo Y, Takano A, et al. Characterization of SEZ6L2 cell-surface protein as a novel prognostic marker for lung cancer. Cancer Sci 2006;97:737-45.

16. An N, Zhao Y, Lan H, et al. SEZ6L2 knockdown impairs tumour growth by promoting caspase-dependent apoptosis in colorectal cancer. J Cell Mol Med 2020;24:4223-32.

17. Wang L, Ling X, Zhu C, et al. Upregulated Seizure-Related 6 Homolog-Like 2 Is a Prognostic Predictor of Hepatocellular Carcinoma. Dis Markers 2020;2020:7318703.

18. Liu J, Lichtenberg T, Hoadley KA, et al. An Integrated

Cite this article as: Luo $\mathrm{X}$, Chen $\mathrm{X}$, Chen S, Gao Q, Yang H, Zhao D. High expression of SEZ6L2 as an independent prognostic Indicator in thyroid carcinoma. Gland Surg 2022;11(2):412-425. doi: 10.21037/gs-22-37
TCGA Pan-Cancer Clinical Data Resource to Drive HighQuality Survival Outcome Analytics. Cell 2018;173:400416.e11.

19. Qiu WQ, Luo S, Ma SA, et al. The Sez6 Family Inhibits Complement by Facilitating Factor I Cleavage of C3b and Accelerating the Decay of C3 Convertases. Front Immunol 2021;12:607641.

20. Liu Y, Zhu D, Xing H, et al. A 6-gene risk score system constructed for predicting the clinical prognosis of pancreatic adenocarcinoma patients. Oncol Rep 2019;41:1521-30.

21. Bornstein S, Schmidt M, Choonoo G, et al. IL-10 and integrin signaling pathways are associated with head and neck cancer progression. BMC Genomics 2016;17:38.

22. Gorlov IP, Meyer P, Liloglou T, et al. Seizure 6-like (SEZ6L) gene and risk for lung cancer. Cancer Res 2007;67:8406-11.

23. Nishioka M, Kohno T, Takahashi M, et al. Identification of a 428-kb homozygously deleted region disrupting the SEZ6L gene at 22q12.1 in a lung cancer cell line. Oncogene 2000;19:6251-60.

(English Language Editor: C. Mullens) 